\title{
Adaptive individual handling for neural network synthesis
}

\author{
Pavel Vařacha ${ }^{1, a}$ \\ ${ }^{1}$ Tomas Bata University in Zlín, Faculty of Applied Informatic, nám. T. G. Masaryka 5555
}

76001 Zlín, Czech Republic

\begin{abstract}
Neural Network Synthesis is an algorithm capable of creating and learning and artificial neural networks as well as optimizing their structures and connections. The method is based on Analytic Programming and asynchronous implementation of Self-Organising Migration Algorithm. Such approach already recorded several successful application considering practical casers of modelling and simulation. This results vindicate efforts for its further development. This paper explores a possibility to make it more effective by adaptive individual handling. The main idea is an intelligent control the process based on complexity of processed neural network structure.

(C) 2016 The Authors. Published by Elsevier Ltd.

Peer-review under responsibility of DAAAM International Vienna.
\end{abstract}

Keywords: neural; network; synthesys; SOMA; adaptive; strategy; structural; optimization

\section{Introduction}

Neural Network Synthesis (ANN synthesis) is an algorithm capable of creating and learning and artificial neural networks as well as optimizing their structures and connections. To describe this method in all details would significantly exceed a possible extend of this paper. Nevertheless ANN synthesis mechanisms are very well elaborated in papers [1] and [2]. An interested reader is respectfully asked to study this previous publications as are referred at the end of the paper.

ANN synthesis is based on Analytic Programming (AP) and asynchronous implementation of Self-Organizing Migration Algorithm (SOMA). Important facts about AP which are necessary for basic understanding of an idea proposed in the paper as an adaptive individual handling are described in chapters 2. And 3. For better understanding of SOMA, please, refer paper [3] or [4].

This paper explores a possibility to make it more effective by adaptive individual handling. The main idea is an intelligent control of the ANN synthesis based on complexity of processed neural network structure.

\section{Analytic programming}

The main principle (core) of AP is based on a discrete set handling (DSH) (Fig. 1). DSH shows itself as a universal interface between the EA and the symbolically solved problem. This is why AP can be used almost by any evolutionary algorithm. In the case of ANN synthesis the algorithm is SOMA.

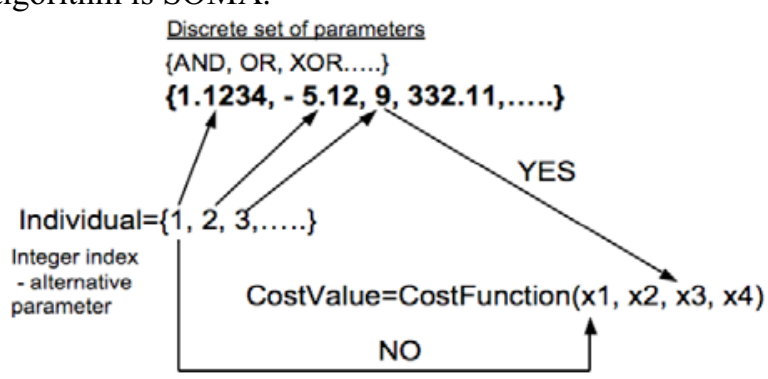

Figure 1. DSH principle.

Briefly stated, in AP, individuals consist of nonnumerical expressions (operators, functions,...) which are represented within the evolutionary process by their integer indexes. Each index then serves as a pointer into the set of expressions and AP uses it to synthesize the resulting function-program for the Cost Function evaluation.

$=$ All simple functions and operators are in the so called General Function Set (GFS) divided into groups according to the number of arguments which can be inserted during the evolutionary process to create subsets GFS3, GFS2...GFS0.

\footnotetext{
${ }^{a}$ Corresponding author: varacha@fai.utb.cz
} 
Table 1. Example of GFS and its subsets.

\begin{tabular}{|c|c|}
\hline GFS Degree & Contains \\
\hline GFSall & $\begin{array}{c}\mathrm{f}(\mathrm{x} 1, \mathrm{x} 2, \mathrm{x} 3),+,-,{ }^{*}, / \text {, Power, Abs, Round, } \\
\text { Sin, Cos, t, K, } \tau, 1,2\end{array}$ \\
\hline GFS3 & $\mathrm{f}(\mathrm{x} 1, \mathrm{x} 2, \mathrm{x} 3)$ \\
\hline GFS2 & $+,-,{ }^{*}, /$, Power \\
\hline GFS1 & Abs, Round, Sin, Cos \\
\hline GFS0 & $\mathrm{t}, \mathrm{K}, \tau, 1,2$ \\
\hline
\end{tabular}

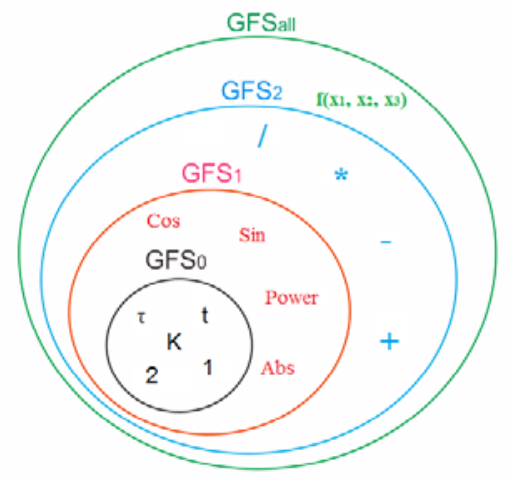

Figure 2. GFS subsets hierarchy.

The functionality of AP can be seen in the specific example in Figure 3:

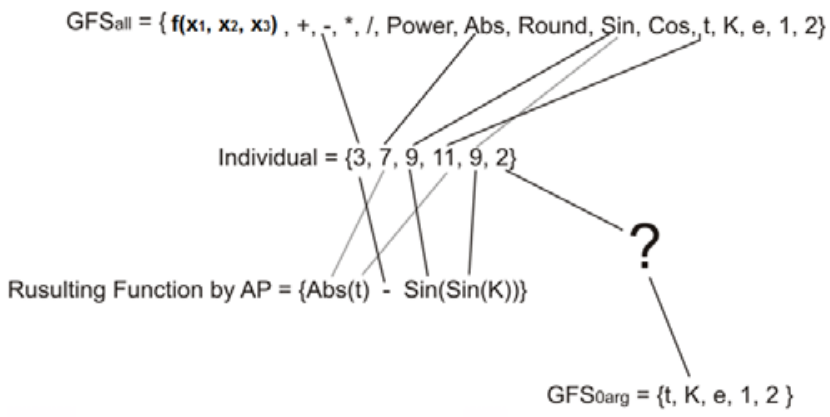

Figure.3. Main principles of AP.

The individual consists of 6 arguments (indices, pointers to GFS). The first index is 3 , meaning that it is taken from the set of functions $\mathrm{GFS}_{\text {all }}$. The function minus has two arguments; therefore indexes 7 and 9 are arguments of minus.

$$
6+7
$$

Index 7 is then replaced by $A b s$ and index 9 by $\operatorname{Sin}$.

$$
A b s+\operatorname{Sin}
$$

$A b s$ and $\operatorname{Sin}$ are one-argument functions. Then, index 9 follows index 11, which is replaced by $t$.

$$
\operatorname{Abs}(t)+\operatorname{Sin}
$$

$\operatorname{Sin}$ is also a one-argument function. Then, after index 11, the individual takes index 9, which is replaced by $\operatorname{Sin}$ and this $\operatorname{Sin}$ becomes an argument of the previous $\operatorname{Sin}$.

$$
\operatorname{Sin}(\operatorname{Tan})+\operatorname{Sin}(\operatorname{Sin}(
$$

The last index is 2 , but in this case there is the function Plus. Plus needs two arguments to work properly. AP will not allow this, as there is not any other free pointer to be used as the argument. Instead of Plus, AP will jump into the subspace, in this case directly to the $\mathrm{GFS}_{0 \text { arg. In }}$ the $\mathrm{GFS}_{0 \text { arg }}$ it finds the second element, which is $K$. And by doing so, we get (5).

$$
\operatorname{Abs}(t)+\operatorname{Sin}(\operatorname{Sin}(K))
$$

The number of pointers actually used from an individual before the synthesized expression is closed is called depth. This example is based on the relevant and previously published work in.

\section{Neural network synthesis}

The previous chapter described basic concepts of AP. Such concepts can be easily employed to synthetize different ANN structures as can be seen from Figure 4. This process of structural synthesis as well as ANN learning is well described for example in [3] and [4].
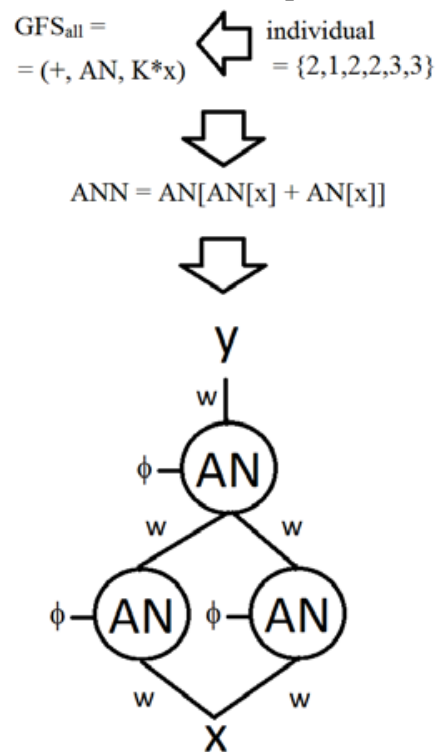

Figure 4. An Example of ANN synthesis.

\section{Experiment designed for adaptive individual handling}

Processes described in chapters 2 . and 3 . have to be optimized by a specific evolutionary algorithms. In case of ANN synthesis it basically means to try variously complex ANN structures (individuals in AP) to improve them in evolutionary way. In place of such evolutionary algorithm ANN synthesis commonly employ SOMA. An algorithm which is well described in [3] and [4]. However SOMA is not anyhow adaptive to coop with various lengths of individuals in AP. SOMA simply treat all such individuals as an individual of constant length (typically 100). For this paper one control parameter of SOMA was chosen to improve this behavior.

An adaptive individual handling proposed in this paper aim to influence control parameter of SOMA named PRT to improve its ability to deal with different lengths of individuals. For each individual SOMA will used different setting of PRT bases on length of a solved individual itself.

Commonly, SOMA is set on PRT $=0.1$. In contradiction this paper proposes adaptive strategy so PRT will differ from individual to individual PRT $=1 /$ depth.

In order to statistically evaluate this new adaptive handling approach the function approximation problem was chosen as an aim of the experiment. The function (6) 
proposed by [5] as an appropriate approximation benchmark was chosen to be approximated by the ANN.

$$
y=x_{i}^{5}-2 x_{i}^{3}+x_{i}
$$

where $x_{i}$ is in $<-1$, by the step $0.04,1>$

Figure 5 (automatically generated by ANN synthesis software) shows an example of synthetized ANN approximating (6). The difference between the ANN and (7) is depicted as a red area which could be minimized by the process of synthesis. An goal of ANN synthesis in this case can be simply described as founding smallest possible ANN able to approximate given function. Simplicity of such function is purely purposeful here as the main goal is not test ability of ANN synthesis to solve complicated tasks but to test how the method improve while applying proposed adaptive strategy.

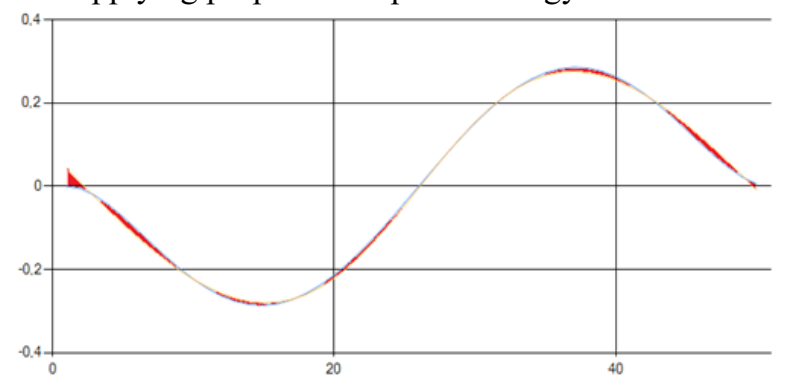

Figure 5. Approximation of (7) by synthetized ANN.

AP was executed 100 times (physically on 8 cores of the Super Micro Server) to produce an ANN with the RMSD $<0.005$. The main intention was to find such an ANN which met this condition and which simultaneously used as few AN as possible. All SOMA's control parameters required for repetition of this experiment are described in following tables. The setting of Asynchronous SOMA used as the EA for AP can be seen in Table 2. and SOMA setting used for ANN learning in Table 3 .

Table 2. Setting of SOMA used as EA for AP.

\begin{tabular}{|c|c|}
\hline Number of Individuals & 48 \\
\hline Individual Parameters & 100 \\
\hline Low & 0 \\
\hline High & 3 \\
\hline PathLength & 3 \\
\hline Step & 0,11 \\
\hline PRT & Based on experiment \\
\hline Divergence & 0.01 \\
\hline Period & 1 \\
\hline
\end{tabular}

Table 3. Setting of SOMA used to optimize Kn.

\begin{tabular}{|c|c|}
\hline Number of Individuals & number ofKn*0.5 (at least 10) \\
\hline Individual Parameters & 100 \\
\hline Low & -10 \\
\hline High & 10 \\
\hline PathLength & 3 \\
\hline Step & 0,11 \\
\hline
\end{tabular}

\begin{tabular}{|c|c|}
\hline PRT & $1 / \mathrm{K}_{\mathrm{n}}$ \\
\hline Divergence & 0.01 \\
\hline Period & 6 \\
\hline
\end{tabular}

Based on experiment setting PRT which SOMA used to optimize $\mathrm{Kn}$ is set either conservatively to $\mathrm{PRT}=0.1$ or adaptively PRT $=1 /$ depth

\section{Results}

The adaptive strategy for individual handling consists in replacement of the static PRT value by the value which inversely depends on depth of a currently operated individual.

Table 4. PRT strategy for individual handling.

\begin{tabular}{|c|c|c|}
\hline & PRT $=1 /$ depth & PRT $=0.1$ \\
\hline $\begin{array}{c}\text { Average time } \\
\text { needed for synthesis }\end{array}$ & $194 \mathrm{~s}$ & $373 \mathrm{~s}$ \\
\hline $\begin{array}{c}\text { Average number of } \\
\text { used AN }\end{array}$ & 9 & 13 \\
\hline
\end{tabular}

A total of $1,189,870$ evaluations of AP individual fitness were completed during 100 AP executions while the PRT was set to 0.1 and the separate SOMA run was performed for all of them to set their Kn value. Without the adaptive PRT, AP was able to find an optimal ANN in only 1 case in comparison with 4 successful cases in the original experiment.

\section{Conclusion}

ANN synthesis already recorded several successful application considering practical casers of modelling and simulation [6] - [9]. It was also applied on large set of widely recognized benchmark functions [10], [11] with respect to the function approximation, prediction and problems. This results vindicate efforts for its further development.

Obtained results of experiment considered in this paper proves an ability of proposed adaptive individual handling to further improve ANN synthesis. It can significantly improve ANN synthesis ability to overshadow concurrent method of ANN optimization as are [12] - [17].

The method of adaptive individual handling proved in this paper are going to be applied on a practical industrial example and comparison of the improved ANN synthesis is to be subjected by a future study.

\section{Acknowledgements}

This work was supported by the Ministry of Education, Youth and Sports of the Czech Republic within the National Sustainability Programme project No. LO1303 (MSMT-7778/2014). 


\section{References}

1. Zelinka I., Vařacha P., Oplatková Z., Volná, E. 12th International Conference on Soft Computing. Czech Republic, VUT Brno, 25-30 (2006)

2. Vařacha P. Recent Researches in Automatic Control. Montreux : WSEAS Press, 377-382 (2011)

3. Šenkerik R., Zelinka I., Davendra D., Oplatkova Z. Computers \& Mathematics with Applications, 60, Issue 4, 1026-1037, ISSN 0898-1221, doi 10.1016/j.camwa.2010.03.059. (2010)

4. Šenkeř́ik R. Oplatkova Z., Zelinka I, Davendra D., Mathematical and Computer Modelling, ISSN 08957177, 10.1016/j.mcm.2011.05.030 (Available online 27 May 2011)

5. Var̆acha P., Zelinka I. IEEE Proceedings 7th International Conference Computer Information Systems and Industrial Management Applications. Ostrava : IEEE Computer Society, 99-100 (2008)

6. Prechelt L., Proben1-A Set of Neural Network Benchmark Problems and Benchmarking Rules, Universität Karlsruhe, Germany (1994)

7. Mangarianm O.L., Wolberg W.H., Cancer diagnosis via linear programming, SIAM News, 23, Number 5, , p. 1-18 (1990)

8. Král E., Dolinay V., Vašek L., Vařacha P. 14th WSEAS International Conference on Systems. Latest Trands on Systems. 2, Rhodes, WSEAS Press (GR) . 657-659 (2010)

9. CHRAMCOV, Bronislav. Recent Researches in Automatic Control. Montreux: WSEAS Press, 346-351 (2011)

10. Zelinka I., Studies in Fuzziness and Soft Computing, New York : Springer-Verlag, (2004)

11. Koza J. R., Genetic Programming, MIT Press, ISBN 0-262-11189-6 (1998)

12. Jui-Yu W., MIMO CMAC neural network classifier for solving classification problems, Applied Soft Computing, 11, Issue 2, The Impact of Soft Computing for the Progress of Artificial Intelligence, p. 2326-2333 (2011)

13. Falco D.I., Cioppa E., Tarantino, Discovering interesting classification rules with genetic programming, Applied Soft Computing 1, p. 257-269 (2002)

14. Brameier M., Banzhaf W., A comparison of linear genetic programming and neural networks in medical data mining, IEEE Transactions on Evolutionary

15. Turner et al., Grammatical Evolution of Neural Networks for Discovering Epistasis among Quantitative Trait Loci Evolutionary Computation, Machine Learning and Data Mining in Bioinformatics Book Series Title: Lecture Notes in Computer Science Publisher: Springer Berlin / Heidelberg, 86 - 97 (2010)

16. Vonk E., Jain L.C., Johnson R.P Advances in Fuzzy Systems - Applications and Theory, 14, World Science, ISBN: 981-02-3106-7 (1997)

17. Koza J. R. et al. Genetic Programming III; Darwinian Invention and problem Solving, Morgan Kaufmann Publisher, (1999) 